Provided for non-commercial research and education use. Not for reproduction, distribution or commercial use.

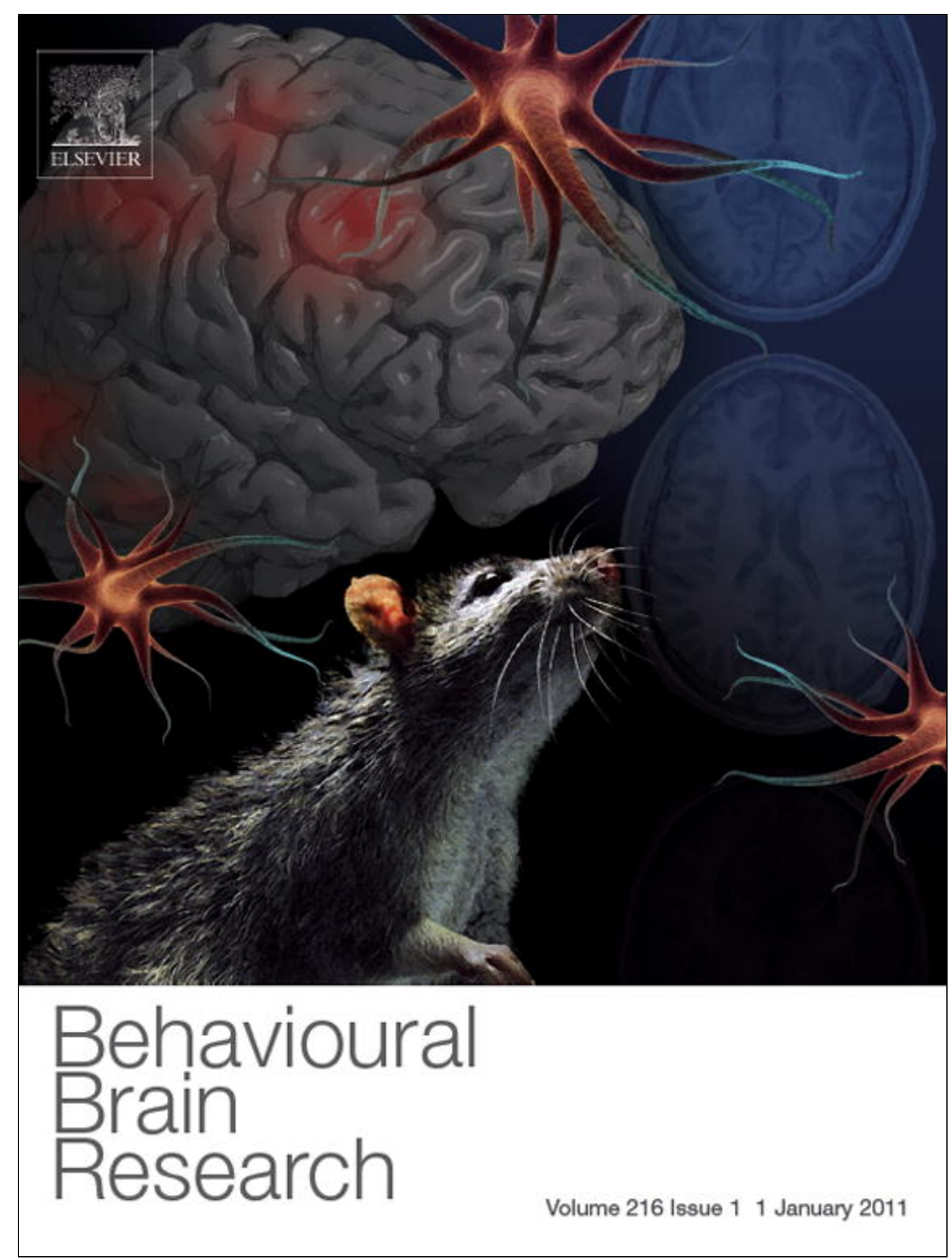

(This is a sample cover image for this issue. The actual cover is not yet available at this time.)

This article appeared in a journal published by Elsevier. The attached copy is furnished to the author for internal non-commercial research and education use, including for instruction at the authors institution and sharing with colleagues.

Other uses, including reproduction and distribution, or selling or licensing copies, or posting to personal, institutional or third party websites are prohibited.

In most cases authors are permitted to post their version of the article (e.g. in Word or Tex form) to their personal website or institutional repository. Authors requiring further information regarding Elsevier's archiving and manuscript policies are encouraged to visit:

http://www.elsevier.com/copyright 
Research report

\title{
Lack of cyclin D2 impairing adult brain neurogenesis alters hippocampal-dependent behavioral tasks without reducing learning ability
}

\author{
Paulina Jedynak $^{\mathrm{a}}$, Piotr Jaholkowski ${ }^{\mathrm{a}}$, Grazyna Wozniak ${ }^{\mathrm{a}}$, Carmen Sandi ${ }^{\mathrm{b}}$, Leszek Kaczmarek ${ }^{\mathrm{a}}$, \\ Robert K. Filipkowski ${ }^{c, *}$
}

a Laboratory of Neurobiology, Nencki Institute of Experimental Biology, Polish Academy of Sciences, 3 Pasteur St., 02-093 Warsaw, Poland

${ }^{\mathrm{b}}$ Laboratory of Behavioral Genetics, Brain Mind Institute, École Polytechnique Fédérale de Lausanne (EPFL), CH-1015 Lausanne, Switzerland

${ }^{\mathrm{c}}$ Department of Biological Psychology, University of Finance and Management in Warsaw, 55 Pawia St., 01-030 Warsaw, Poland

\section{A R T I C L E I N F O}

\section{Article history:}

Received 19 July 2011

Received in revised form 31 October 2011

Accepted 5 November 2011

Available online $\mathrm{xxx}$

\section{Keywords:}

Adult neurogenesis

Hippocampal formation

Hippocampal tasks

Knockout mice

Rodent-specific behaviors

\begin{abstract}
A B S T R A C T
The exact function of the adult brain neurogenesis remains elusive, although it has been suggested to play a role in learning and memory processes. In our studies, we employed cyclin D2 gene knockout (cD2 KO) mice showing impaired neurogenesis as well as decreased hippocampal size. However, irrespectively of the genetic background of $\mathrm{CD} 2 \mathrm{KO}$ mice, this phenotype resulted in neither deficits in the hippocampal-dependent learning ability nor the memory formation. In the present study, cD2 KO mice and control littermates were subjected to hippocampal-dependent behavioral tests with little or no learning component. The knockout mice showed significant impairment in such species-typical behaviors as nest construction, digging, and marble burying. They were building none or poorer nests, digging less robustly, and burying fewer marbles than control mice. Such impairments were previously described, e.g., in animals with hippocampal lesions. Moreover, $\mathrm{CD} 2 \mathrm{KO}$ animals were also more active in the open field and automated motility chamber as well as showed increased explorative behavior in IntelliCage. Both increased motility and explorative behaviors were previously observed in hippocampally lesioned animals. Finally, cD2 KO mice showed normal sucrose preference, however starting from the second exposure to the sweetened solution, while control animals displayed a strong preference immediately. Presented results suggest that either morphological abnormalities of the hippocampal formation or adult brain neurogenesis impairment (or both) alter hippocampal-dependent behaviors of mutant mice without influencing learning abilities. These results may also suggest that adult brain neurogenesis is involved in species-typical behaviors.
\end{abstract}

(C) 2011 Elsevier B.V. All rights reserved.

\section{Introduction}

Cyclin D2 knockout (cD2 KO) mice show greatly impaired adult brain neurogenesis $[1,2]$ most likely because cyclin D2 is the only one cyclin D present in the adult neuronal precursors. The lack of new neurons and most-likely disturbed cell cycle throughout the development of these mice leads to reduced size of several brain structures including smaller hippocampal formation. This particular structure was shown to be smaller by $28 \%$ in mutant mice [2]. Surprisingly, cD2 KO mice showed no cognitive deficits in a battery of hippocampal-dependent learning and memory tasks including, e.g., fear conditioning [1]. They also performed as well as their control siblings in several sensorimotor and emotionality tests.

According to the previous studies, animals with hippocampal lesions show impairments in spontaneous behaviors typical for

\footnotetext{
* Corresponding author. Tel.: +48 668403448; fax: +48 225365412.

E-mail address: filipkowski@vizja.pl (R.K. Filipkowski).
}

small rodents as well as alterations of their locomotor activity and exploration pattern (see below). Our aim was to investigate whether these, non-cognitive, behaviors were also altered in cD2 $\mathrm{KO}$ mice with both impaired adult brain neurogenesis and reduced hippocampal size.

Herein, we employed a number of behavioral tasks which were shown to depend on the intact hippocampal formation. Particularly, animals with electrolytic or neurotoxic lesions or ischemicallydamaged hippocampal formation showed impaired nest building, digging and marble burying conducted in semi-natural conditions [3-7]. Animals with affected hippocampus also showed higher activity and decreased freezing, but on the other hand, lower explorative behavior $[6,8]$. In the present study, the groups of $\mathrm{CD} 2 \mathrm{KO}$ mice and their control siblings were assessed in tests of speciestypical and motor behaviors as well as in both cue and context fear conditioning.

Adult neurogenesis has been implicated in learning and memory phenomena because the hippocampal formation had been as well considered as pivotal for those functions. However this 
structure is also engaged in a number of species-typical behaviors. Our present results may suggest that adult brain neurogenesis could be considered in the latter ones.

\section{Materials and methods}

\subsection{Animals}

Mice: Cyclin D2 mutant mice were generated before $[9,10]$ and backcrossed into C57BL/ 6 background over 12 times in our laboratory. The line was then kept as cyclin D2 heterozygotes ( $+/-$ ) and their homozygous progeny, $-1-(\mathrm{cD} 2 \mathrm{KO})$ and $+/+(\mathrm{WT})$ littermates, were used in all experiments. The animals were kept under a natural light/dark cycle in Plexiglas cages with water and food provided ad libitum. To minimize animal suffering, the rules established by the First Warsaw Ethica Committee on Animal Research and based on the Animal Protection Act of the Polish Republic were strictly followed. The animals were from 2.5 to 5 months old. The age of animals was carefully matched between WT and CD2 KO mice within each group and is always indicated. Experimenters were always unaware of the genotype of the mice. Each behavioral experiment was performed using separate group of mice; only in case of the open field followed by sucrose consumption test, the same animals were employed (following 12-days break). Weighing of the hippocampal formation was performed in 3-4 months old WT $(n=7)$ and CD2 KO mice $(n=8)$.

\subsection{BrdU detection and Nissl staining}

Dividing cells were labeled in 3 months old WT $(n=5)$ and cD2 KO $(n=5)$ female mice by intraperitoneal injection of BrdU $(50 \mathrm{mg} / \mathrm{kg}$ body weight, Sigma, prepared in PBS at $\mathrm{pH}$ 7.4) for two consecutive days, twice daily, $2 \mathrm{~h}$ apart, and killed $24 \mathrm{~h}$ after the last injection. BrdU detection was performed as described elsewhere [1] with modifications - Fluoromount $\mathrm{G}$ (Southern Biotechnology) was used for nuclear staining and mounting. Sections were $50 \mu \mathrm{m}$ thick, 4-6 sections per animal were chosen distanced $300 \mu \mathrm{m}$ from each other. Nissl staining was performed with sections placed on the gelatin-coated slides and dried overnight. Slides were put directly into $0.1 \%$ cresyl violet solution for $5 \mathrm{~min}$, rinsed in distilled water and dehydrated in ethanol solutions and xylene, and coverslipped with Entellan (Merck Chemicals, Poland).

\subsection{Apparatus and procedures}

\subsubsection{Tests with learning components}

2.3.1.1. Cue and context fear conditioning. Female mice ( 3 months old; $\mathrm{cD} 2 \mathrm{KO}$, $n=8$; WT, $n=10$ ) were subjected to the fear conditioning procedure for 3 days, as described before $[11,12]$ with modifications. On day 1 , mice were placed in the conditioning chamber (Med Associates Inc., VT, USA) and received five pairings between a tone $(20 \mathrm{~s}, 85 \mathrm{~dB}, 5 \mathrm{kHz})$ and a co-terminating shock $(1 \mathrm{~s}, 0.7 \mathrm{~mA})$. The inter-trial interval was $60 \mathrm{~s}$, and the first tone presentation commenced $180 \mathrm{~s}$ after the mouse was placed into the chamber (box A). The chamber was cleaned with $70 \%$ ethanol between each mouse. On day 2 , the procedure and context were changed in several ways to test conditioned fear of the tone CS in the absence of contextual cues associated with shock (box B). The grid floor was covered with a white Plexiglas plate; the ventilation fan, the light in the box and in the experimental room were all off; chambers were cleaned with $1 \%$ acetic acid between runs. Each mouse was placed into the chamber for $5.5 \mathrm{~min}$. The tone was presented twice for $20 \mathrm{~s}$ at 120 and $290 \mathrm{~s}$ into the session. No shocks were administered. Freezing was scored during the first and second tone presentation (tone-elicited freezing). On day 3 , mice were tested for conditioned fear of the training context (box A). The testing procedure and context were identical to those used on day 1 , except that the CS and US were not presented. Mice were placed into the chamber for $4 \mathrm{~min}$ and freezing was scored. Freezing behavior was analyzed using VideoFreeze 2.5.4.0 software.

\subsubsection{Species-typical behaviors}

2.3.2.1. Digging. The test was performed as previously described [6]. Female mice, 4-5 months old ( $\mathrm{CD} 2 \mathrm{KO}, n=8 ; \mathrm{WT}, n=9$ ) were placed in a clean $36.5 \mathrm{~cm} \times 20.7 \mathrm{~cm} \times 14.0 \mathrm{~cm}$ cages with approximately $5 \mathrm{~cm}$ thick standard wooden bedding. After each animal testing the bedding was flattened, firmed and reused. Test duration was $210 \mathrm{~s}$. The latency to start digging, the number of digging bouts and the total duration of digging were recorded. Digging was defined as coordinated movements of fore- or hindlimbs that displace the substrate.

2.3.2.2. Marble burying. The test was performed as previously described [13] Male mice, 4-5 months old ( $\mathrm{CD} 2 \mathrm{KO}, n=11$; WT, $n=8$ ) were placed in a clean $42.5 \mathrm{~cm} \times 26.6 \mathrm{~cm} \times 15.5 \mathrm{~cm}$ cages and 12 glass marbles of $15 \mathrm{~mm}$ in diameter were placed in two rows in a regular pattern on the surface, evenly spaced, each about $4 \mathrm{~cm}$ apart on the surface of approximately $5 \mathrm{~cm}$-thick standard wooden bedding. Test duration was $30 \mathrm{~min}$. The number of marbles buried within the bedding (to at least $2 / 3$ of their depth) was counted.
2.3.2.3. Nest construction. The nest building ability was measured as described before [14]. Male mice, 2.5-3 months old ( $\mathrm{CD} 2 \mathrm{KO}, n=9$; WT, $n=9$ ) were housed individually for at least 1 week in $36.9 \mathrm{~cm} \times 15.6 \mathrm{~cm} \times 13.2 \mathrm{~cm}$ cages with standard bedding. A standard piece of paper towel $(23 \mathrm{~cm} \times 23 \mathrm{~cm})$ was provided $3 \mathrm{~h}$ prior to inspection, and the nests were assessed by two observers (inter-rater reliability $>0.9$, weighted Cohen's kappa coefficient), using the following scoring system: $0-$ no nest, 1-primitive flat nest (pad-shaped, consist of a flat paper tissue which slightly elevates a mouse above the bedding), 2-more complex nest (including warping and biting the paper towel), 3-complex accurate cup-shaped nests (with shredded paper interwoven to form the walls of the cup), 4-complex hooded nest, with walls forming a ceiling so the nest becomes a hollow sphere with one opening. The average score from the two observers was used for future analysis.

2.3.2.4. Sucrose-water consumption. During the two dark phases (session 1 and 2 one week apart), male mice, $3-4$ months old (cD2 KO, $n=14$; WT, $n=13$ ) were singlecaged in the $36.5 \mathrm{~cm} \times 20.7 \mathrm{~cm} \times 14.0 \mathrm{~cm}$ cages with 2 bottles containing tap water or $1 \%$ sucrose solution. For the light phases mice were returned to their home cages. Bottles were weighted before and after the first and second dark phase. If a leakage occurred (amount of liquid disappeared from the bottle was more than double standard deviation of the mean), such result was excluded; finally, the results from 13 (cD2 KO) and 9 (WT) animals were analyzed. Experiment was repeated exactly the same way using female mice (3-4 months old, $\mathrm{cD} 2 \mathrm{KO}, n=16$; WT, $n=13)$, and the final numbers of animals analyzed were $12(\mathrm{cD} 2 \mathrm{KO})$ and 9 (WT).

\subsubsection{Motor and activity tests}

2.3.3.1. Motility chamber. For activity and motility measurements, 4-5 months old female mice ( $\mathrm{CD} 2 \mathrm{KO}, n=10$; WT, $n=10$ ) were used. Tests were conducted in $43 \mathrm{~cm} \times 43 \mathrm{~cm} \times 18 \mathrm{~cm}$ clear Plexiglas automated activity chamber (Opto-Varimex 3 unit, Columbus Instruments, OH, USA) equipped with 15 infrared beams on each axis in the horizontal plane to detect horizontal movement. Using the ATM3 AutoTrack System software the raw count of beam breaks and the actual position of the subject were converted into: distance travelled, horizontal counts (any beam broken at any time), ambulatory counts (a subgroup of horizontal counts registered only when different beams were broken, while rapidly breaking the same beam did not register additional counts), ambulatory time, small stereotypic movement (scratching, digging, grooming, etc. measured as a repetitive single beam counts) number of bursts of stereotypic movement (groups of stereotypic counts separated by $1 \mathrm{~s}$ ), resting time (no beam counts registered). The test lasted for $20 \mathrm{~min}$ and was scored after the first 5, 10, and 20 min time-lapses.

2.3.3.2. Open field. The apparatus was a wooden floor $(50 \mathrm{~cm} \times 50 \mathrm{~cm})$ surrounded by walls ( $34 \mathrm{~cm}$ high), and divided into $10 \mathrm{~cm} \times 10 \mathrm{~cm}$ squares. The open field is potentially sensitive to both activity and exploration, and to emotionality, which tends to suppress the expression of the former. Our aim was to highlight activity and exploration, not anxiety, so the normal room illumination was used, rather than extra bright lights, and the maze was painted grey, not white. Animals' behavior was monitored by a video camera placed above the center of the apparatus. The apparatus arena was virtually divided into 25 identical square zones which were cumulated in 3 zones: thigmotaxic, intermediate $(10 \mathrm{~cm}$ wide each), and center $(10 \mathrm{~cm} \times 10 \mathrm{~cm})$. Male mice, $3-4$ months old $(\mathrm{cD} 2 \mathrm{KO}, n=13$; WT, $n=14)$ were put individually in one corner of the open field facing the wall and were allowed to explore freely for $10 \mathrm{~min}$. The floor of the apparatus was cleaned with $5 \%$ ethanol after each session. Data were analyzed using EthoVision 3.1. system (Noldus Information Technology, The Netherlands), and the following parameters were counted: total distance moved and frequency of presence in zones as a total number of times the animal has been in the virtual zones.

2.3.3.3. IntelliCage. Male mice (cD2 KO, $n=20$; WT, $n=20 ; 3-4$ months old) were tested in an automated apparatus, an IntelliCage system (NewBehavior AG, Switzerland) as described before [1]. The animals of the same genotype were placed in groups of 10 per cage. The explorative behavior was measured during $48 \mathrm{~h}$ of exploration of the cage when all doors were opened and mice had unlimited access to the drinking bottles and food. The number of visits in the corners and visits duration were measured, the results more than twice the standard deviation above or below the means were excluded; finally, the results from 16 (cD2 KO) and 17 (WT) animals were analyzed.

2.3.3.4. Accelerating rotarod. Male mice ( $\mathrm{CD} 2 \mathrm{KO}, n=14$; WT, $n=14 ; 3-4$ months old $)$ were submitted to 3 trials on the rotarod (TSE Systems, Inc., MO, USA) with $45 \mathrm{~min}$ rest periods between trials. Starting speed was $4 \mathrm{rpm}$, acceleration was determined by setting the time to reach a maximum speed of $40 \mathrm{rpm}$, which for constant acceleration of $7 \mathrm{rpm} / \mathrm{min}$ is $5 \mathrm{~min}$. The average latency to fall from the rod and the distance travelled were measured.

\subsection{Data analysis and results presentation}

Pairwise comparisons between the WT and cD2 KO groups were performed using ANOVA test for parametric data. When possible, the data were submitted 
to 2- or 3-way repeated measures ANOVA followed by planned comparisons. In cases where the data did not meet the assumptions for ANOVA, the results were transformed using Box-Cox transformation, as advised by the statistical software package used (JMP 8.0.1.) which tests for normality and equality of variance. Nonparametrical Mann-Whitney and Wilcoxon tests, where appropriate, were used where data still violated the assumptions for ANOVA. For parametric data, the mean \pm S.E.M. values are given. For non-parametric data, the median with the interquartile range (IQR, calculated with Microsoft Office Excel 2003) in parentheses are given. The minimal level of significance was $P<0.05$.

\section{Results}

Since in the present study, we employed a novel genetic background $(\mathrm{C} 57 \mathrm{BL} / 6)$ of the transgenic $\mathrm{CD} 2 \mathrm{KO}$ mice while a mixed genetic background was used before $[1,2]$, we first reproduced major previous findings - concerning the impaired adult brain neurogenesis and size of the hippocampal formation as well as simple hippocampal-dependent learning paradigm.

\subsection{Adult brain neurogenesis and size of hippocampal formation}

In cD2 KO mice, in the subgranular layer of the dentate gyrus, we observed decreased number of cells which incorporated BrdU, 0.6 (0.4-1.1, IQR), when compared to WT controls, 24.0 (22.8-24.8, IQR) $(P<0.01$, Mann-Whitney, $U=0)$, Fig. $1 C$ and D. The hippocampal formation of $\mathrm{CD} 2 \mathrm{KO}$ mice also weighted less than in WT $[\mathrm{mg}]: 24.2 \pm 1.0$, and $34.2 \pm 0.7$, respectively $\left(P<0.001, F_{(1,13)}=67.3\right.$, ANOVA), Fig. $1 \mathrm{~A}$ and $\mathrm{B}$.

\subsection{Task with learning components - cue and contextual fear conditioning}

There was no deficit in cue and context fear conditioning when cD2 KO were compared to WT mice. Animals of both genotypes did not freeze during the first day of training before receiving shock. Both CD2 KO and WT mice showed increased cue conditioned freezing in the novel context on day 2 when the tone was presented. 2-way ANOVA of the level of freezing, with genotype and tone presentation as factors, showed no effect of genotype and highly significant effect of tone presentation $\left(F_{(1,16)}=28.0\right.$, $P<0.001)$. Planned comparisons showed higher level of freezing to the tone for both WT $\left(F_{(1,16)}=26.3, P<0.001\right)$ and CD2 KO animals $\left(F_{(1,16)}=6.3, P<0.05\right)$, with no significant difference between WT and CD2 KO mice. During the first tone presentation CD2 KO mice were freezing for [\% of time] $52.1 \pm 5.7$ and WT controls for $57.1 \pm 7.8$, Fig. 1E. During the second tone presentation the level of freezing [\% of time] was $68.1 \pm 8.8$ for CD2 KO mice and $61.9 \pm 9.2$ for WT mice. On day 3, when the animals were retrieved to the familiar cage, again the mice of both genotypes showed significantly increased $(P<0.01$ for WT mice, $P<0.05$, Wilcoxon) context conditioning freezing [\% of time]: $35.2 \pm 4.4$ (cD2 KO mice); $30.9 \pm 6.3$ (WT mice), measured during the first minute in the familiar context, and no difference between the two genotypes was demonstrated $\left(P>0.05, F_{(1,16)}=0.3\right.$, ANOVA) $($ Fig. 1E).

\subsection{Tasks without learning components}

\subsubsection{Species-typical behaviors}

3.3.1.1. Digging. The latencies [s] to start digging were higher for the cD2 KO mice than WT controls: 194.6 (139.0-210.0, IQR) and 73.8 (57.4-93.1, IQR), respectively $(P<0.05$, Mann-Whitney, $U=11.0)$. Mutant mice performed less digging bouts: $0.5(0.0-2.5$, $\mathrm{IQR})$ versus $3.0(3.0-11.0, \mathrm{IQR})$ in control mice $(P<0.05$, MannWhitney $U=11.0)$. They also spent less time digging than control animals [s]: $0.5(0.0-3.1, \mathrm{IQR})$ and $4.6(2.6-10.5, \mathrm{IQR})$, respectively $(P<0.05$, Mann-Whitney, $U=13.0)$, Fig. 2 A.
3.3.1.2. Marble burying. Mutant animals buried significantly less marbles than WT controls: $3.0 \pm 0.5$ and $6.6 \pm 0.8$, respectively $\left(P<0.01, F_{(1,17)}=14.0\right.$, ANOVA $)$, Fig. 2B.

3.3.1.3. Nest construction. WT animals performed better in this task than cD2 KO mice, in that their nests were bigger and better formed. Median and IQR scores were, respectively: 2.5 (1.5-3.0, IQR) and 0.0 (0.0-2.0, IQR) $(P<0.05$, Mann-Whitney, $U=18.5)$, Fig. 2C. Similar results were obtained for an independent group of animals (WT, $n=9,2.0$ (2.0-2.0, IQR); cD2 KO, $n=6,0.0$ (0.0-0.8, IQR); aged 4-6 months; $P<0.05$, Mann-Whitney test, $U=8.5$ ).

3.3.1.4. Sucrose-water consumption. Mice show strong preference for sucrose, which was confirmed by an effect of the treatment $\left(F_{(1,20)}=33.9, P<0.001\right)$. There was also an effect of the session $\left(F_{(1,20)}=15.4, P<0.001\right)$ and the effect of the treatment depended on the session (treatment $\times$ session effect; $F_{(1,20)}=30.8, P<0.001$, ANOVA). Particularly, during the first exposition, cD2 KO mice did not show preference for the sucrose solution in contrary to WT controls. Mutant mice drunk [ml] $5.8 \pm 0.4$ of sucrose, and $5.8 \pm 0.3$ of water $\left(P>0.05, F_{(1,20)}=0.0\right.$, planned comparison). Control animals drunk [ml] $6.4 \pm 0.5$ of sucrose, and $4.5 \pm 0.3$ of water $\left(F_{(1,20)}=6.5\right.$, $P<0.05$, planned comparisons). During the second session, both CD2 KO and WT drunk more sucrose solution than water, and there was no difference between the groups. Mutant mice drunk $[\mathrm{ml}]$ : sucrose $(8.6 \pm 0.6)$, water $\left(5.2 \pm 0.5, F_{(1,20)}=33.1, P<0.001\right.$, planned comparisons), control mice $[\mathrm{ml}]$ : sucrose $(8.9 \pm 0.6)$, water $\left(4.8 \pm 0.4, F_{(1,20)}=32.6, P<0.001\right.$, planned comparisons), Fig. 2D. Female mice (second group, not shown) also show strong preference for sucrose, which was confirmed by an effect of the treatment $\left(F_{(1,19)}=43.7, P<0.001\right)$. There was no effect of the session but the effect of the treatment depended on the session (treatment $\times$ session effect; $F_{(1,19)}=25.0, P<0.001$, ANOVA) and on the genotype (treatment $\times$ genotype effect, $F_{(1,19)}=7.1, P<0.05$, ANOVA). There was also an effect of the genotype, $F_{(1,19)}=4.6$, $P<0.05$, ANOVA). Particularly, during the first exposition, as in case of male mice, $\mathrm{cD} 2 \mathrm{KO}$ mice did not show preference for the sucrose solution in contrary to WT controls. Mutant mice drunk $[\mathrm{ml}] 5.9 \pm 0.5$ of sucrose, and $5.7 \pm 0.2$ of water $\left(P>0.05, F_{(1,20)}=0.0\right.$, planned comparisons), while control animals drunk [ml] $8.3 \pm 0.4$ of sucrose, and $5.2 \pm 0.3$ of water $\left(F_{(1,19)}=21.4, P<0.001\right.$, planned comparisons). During the second session, both cD2 KO and WT drunk more sucrose solution than water. Mutant mice drunk $[\mathrm{ml}]$ : sucrose $(8.0 \pm 0.3)$, water $\left(5.1 \pm 0.6, F_{(1,19)}=22.8, P<0.001\right.$, planned comparisons), control mice [ml]: sucrose $(9.1 \pm 0.4)$, water (4.6 $\pm 0.5, F_{(1,19)}=36.0, P<0.001$, planned comparisons $)$.

\subsubsection{Motor and activity tests}

3.3.2.1. Motility chamber. Mutant mice were significantly more active than WT controls. Distance travelled [m] was longer for cD2 KO $(153.3 \pm 4.1)$ than for WT mice $(133.1 \pm 4.9, P<0.01$, $F_{(1,18)}=10.0$, ANOVA). They also showed higher horizontal movement counts (cD2 KO: $7590 \pm 497$, and WT: $6146 \pm 351, P<0.05$, $F_{(1,18)}=5.6$, ANOVA), ambulatory movement counts (cD2 KO: $5114 \pm 400$, and WT: $4014 \pm 288, P<0.05, F_{(1,18)}=5.6$, ANOVA), and ambulatory movement time [min] (CD2 KO: $15.9 \pm 0.3$, and WT: $14.9 \pm 0.3, P<0.05, F_{(1,18)}=5.9$, ANOVA). Moreover, cD2 KO mice showed shorter time [min] of small stereotypic movement than WT controls $(2.6 \pm 0.1$ and $3.1 \pm 0.2$, respectively, $P<0.01$, $F_{(1,18)}=9.2$, ANOVA), and tendency for decreased number of stereotypic movements bursts, however the difference did not reach significance (114.2 \pm 4.3 , cD2 KO mice; $126.1 \pm 4.2$, WT mice; $P=0.06, F_{(1,18)}=3.9$, ANOVA). Resting time was not significantly different between $\mathrm{CD} 2 \mathrm{KO}$ and WT mice $\left(P>0.05, F_{(1,18)}=2.2\right.$, ANOVA), 

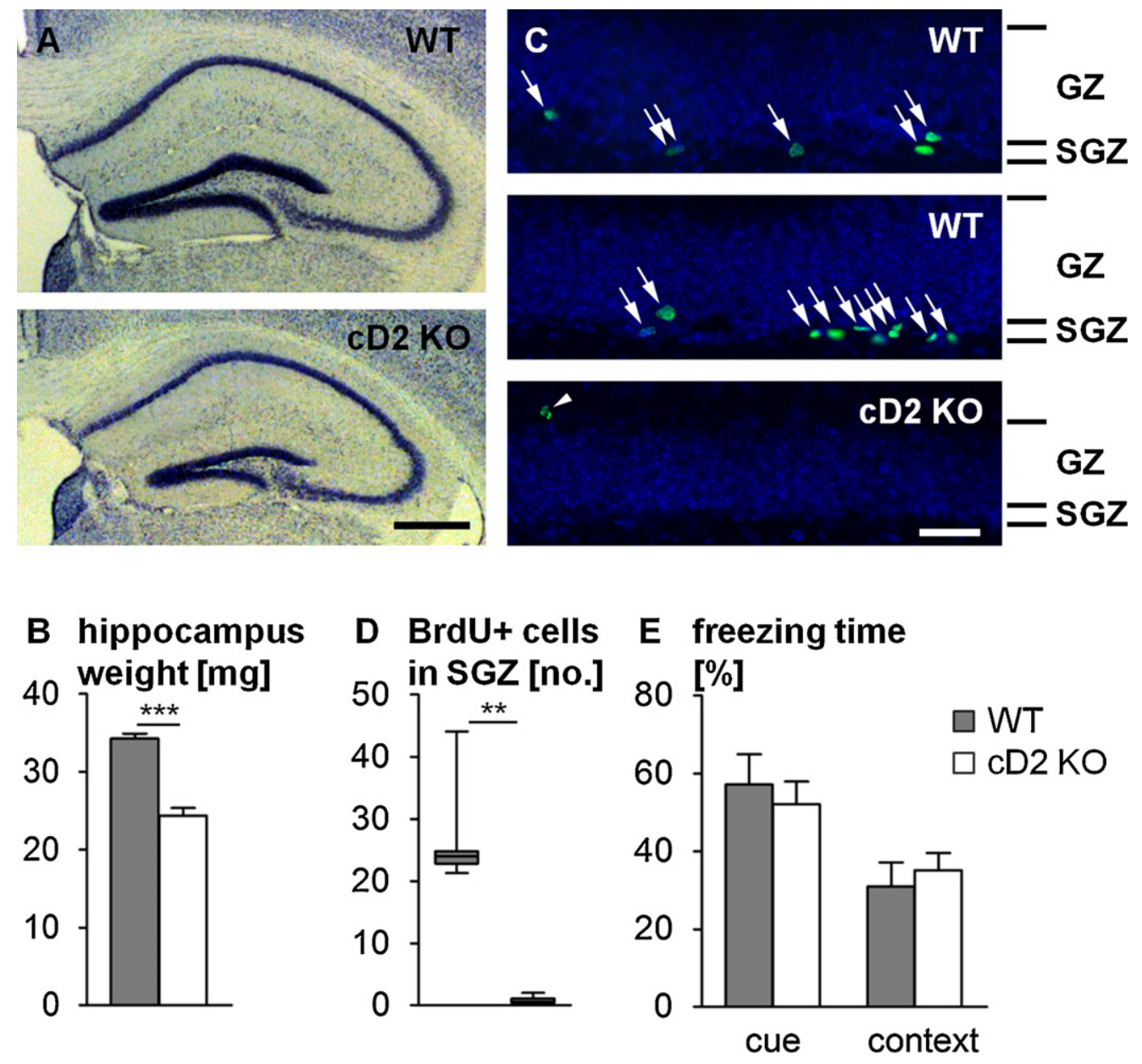

Fig. 1. Mutant $\mathrm{CD} 2 \mathrm{KO}$ mice show reduction in hippocampal size and deficit in adult brain neurogenesis, but no learning alterations. (A, B) Hippocampal formation of cD2 KO mice was significantly smaller than in WT as shown by Nissl staining, scale bar: $500 \mu \mathrm{m}$ (A) and by weighing the structure (B). (C, D) Mutant mice show a profound deficit in adult brain neurogenesis in the subgranular zone (SGZ) of the dentate gyrus. (C) Representative coronal sections of two WT subjects and one cD2 KO mouse showing the granule zone (GZ) cell layer, visualized by nuclear marker (DAPI, blue), stained with proliferation marker BrdU (green, arrows in WT). The number of BrdU-positive cells was almost completely reduced in cD2 KO, and only a few were found scattered around GZ, however, their localization usually suggested non-neuronal nature of these cells (arrowhead); scale bar, $40 \mu \mathrm{m}$. (D) Number of BrdU-positive cells in the subgranular zone of the hippocampal formation. (E) Mutant cD2 KO mice show normal cue and context conditioning memory. Both groups show enhanced freezing when moved to a novel context and when the tone (cue) was presented. In the same context in which animals were trained, both WT and mutant animals displayed increased freezing levels when compared to basal freezing, the first minute of freezing is shown. The results are presented as means \pm S.E.M (B, E) or as median with interquartile range, minimum and maximum (D); $\left(^{* *}\right) P<0.01,\left({ }^{* * *}\right) P<0.001$.

Fig. 3A. All the differences were observed after $20 \mathrm{~min}$, in the earlier time points ( 5 and $10 \mathrm{~min}$ ) differences were not significant.

3.3.2.2. Open field. Mutant cD2 KO mice were significantly more active than WT controls in the open field. Distance travelled [m] was longer for $\mathrm{CD} 2 \mathrm{KO}(58.6 \pm 3.1)$ than for WT mice $(48.0 \pm 2.3$, $P<0.05, F_{(1,25)}=7.7$, ANOVA). They also showed higher frequency of presence in all the zones of the arena when compared to WT controls: $316 \pm 24$, and $240 \pm 16$ crossings, respectively $(P<0.05$, $F_{(1,25)}=5.7$, ANOVA). However, the time spent in any zone did not differ between $\mathrm{CD} 2 \mathrm{KO}$ and control mice, Fig. 3B.

3.3.2.3. Exploration in IntelliCage. Transgenic cD2 KO mice were also more active in IntelliCage system. They visited the corners more frequently when compared to WT controls. An ANOVA of visit numbers revealed a significant effect of the genotype $\left(F_{(1,31)}=7.7\right.$, $P<0.01)$ and of the day $\left(F_{(1,31)}=6.5, P<0.05\right)$. Following the first day of analysis, $\mathrm{CD} 2 \mathrm{KO}$ mice paid more visits $(141.0 \pm 7.3)$ than WT mice $\left(117.5 \pm 9.2 ; F_{(1,31)}=6.8, P<0.05\right.$, planned comparisons $)$. Similarly, during the second day of observation, CD2 KO mice visited the corners more often $(122.0 \pm 5.1)$ than WT control mice $(98.8 \pm 7.8$; $F_{(1,31)}=4.3, P<0.05$, planned comparisons), Fig. 3C. Not only cD2 KO mice visited the corners more often than WT mice, but also they spent more time in the corners. An ANOVA showed a highly significant effect of the genotype $\left(F_{(1,31)}=17.8, P<0.001\right)$. The effect of the genotype depended on the day - (genotype $\times$ day effect, $F_{(1,31)}=5.0$, $P<0.05)$. During the first $24 \mathrm{~h}$ of observation, cD2 KO mice spent more time [ $\mathrm{min}]$ in the corners $(49.5 \pm 5.5)$ than WT control littermates $\left(30.3 \pm 2.0 ; F_{(1,31)}=9.9, P<0.01\right.$, planned comparisons), while during the second $24 \mathrm{~h}$, the difference was even more pronounced - with cD2 KO spending on average $59.6 \pm 7.0 \mathrm{~min}$ in the corners compared to $26.9 \pm 3.0 \mathrm{~min}$ of average visit duration by WT control mice $\left(F_{(1,31)}=18.2, P<0.001\right.$, planned comparisons $)$, Fig. 3C.

3.3.2.4. Accelerating rotarod. Mutant mice did not differ from WT controls neither in latency to fall from the rod [s]: $\mathrm{cD} 2 \mathrm{KO}$ mice, $94.0 \pm 9.5$; WT mice, $77.1 \pm 8.1\left(P>0.05, F_{(1,26)}=1.8\right.$, ANOVA $)$, nor in the distance travelled [m]: $\mathrm{CD} 2 \mathrm{KO}$ mice, $1.6 \pm 0.2$; WT mice, $1.2 \pm 0.2\left(P>0.05, F_{(1,26)}=1.6\right.$, ANOVA $)$. 

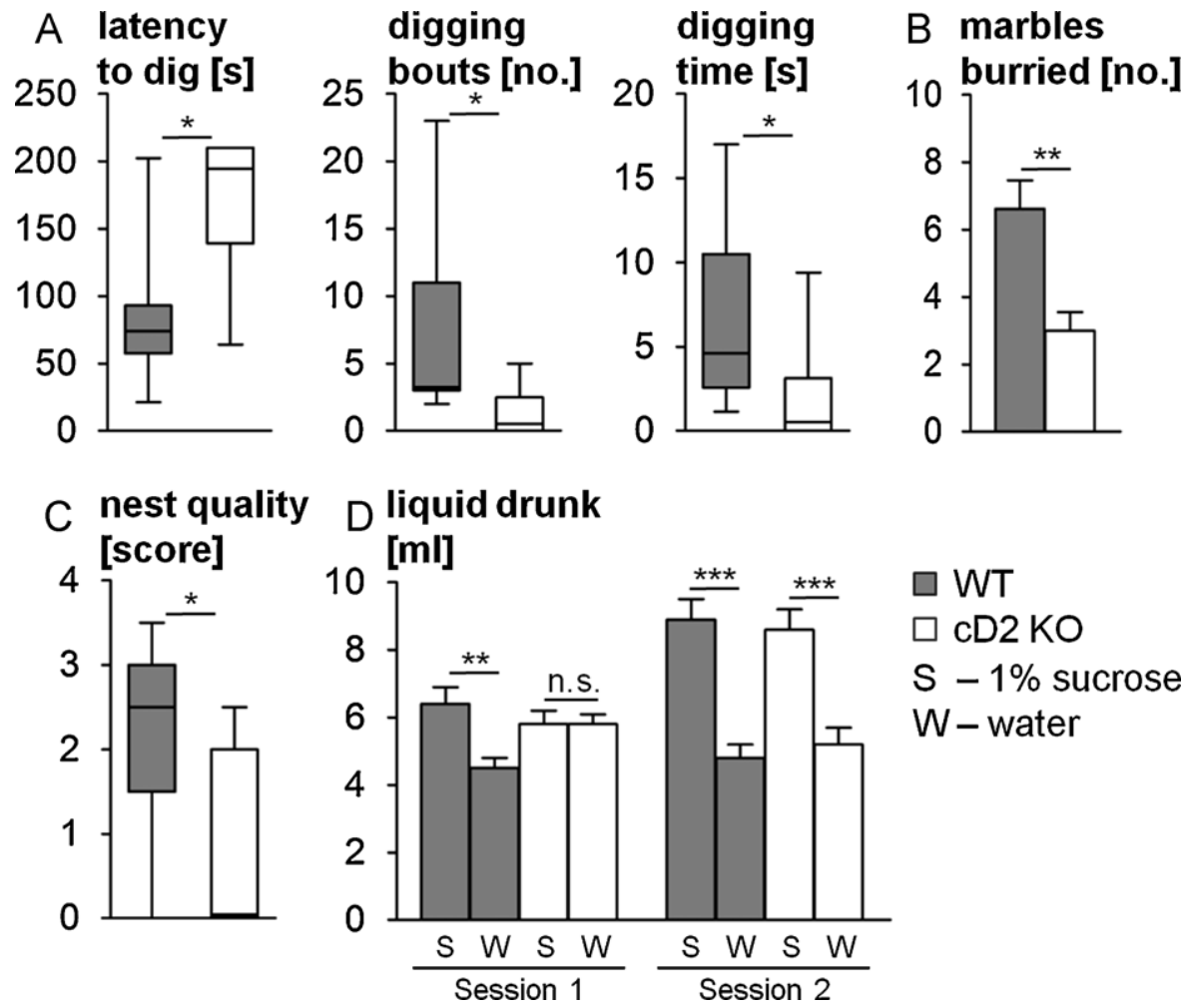

Fig. 2. Mutant mice are impaired in rodent-typical behaviors such as digging, marble burying, and nesting. They did not differ in sucrose consumption, however did not show preference for sucrose during the first exposition to the sweetened water. (A) cD2 KO mice showed increased latency to start digging, performed less digging bouts and spent shorter time digging. (B) Mutant mice buried less marbles than WT controls. (C) CD2 KO animals built no or poorer nests comparing to WT controls. (D) During the first exposition to sucrose (Session 1), cD2 KO mice did not show preference to sweetened water. Control animals showed strong preference from the first exposition to sucrose. During the second exposition to sucrose (Session 2) both cD2 KO and WT animals showed preference to sweetened water. The results are presented as means \pm S.E.M. (B, D) or as median with interquartile range, minimum and maximum $(\mathrm{A}, \mathrm{C}) ;\left({ }^{*}\right) P<0.05,\left({ }^{* *}\right) P<0.01,\left({ }^{* * *}\right) P<0.001$, (n.s.) not significant.
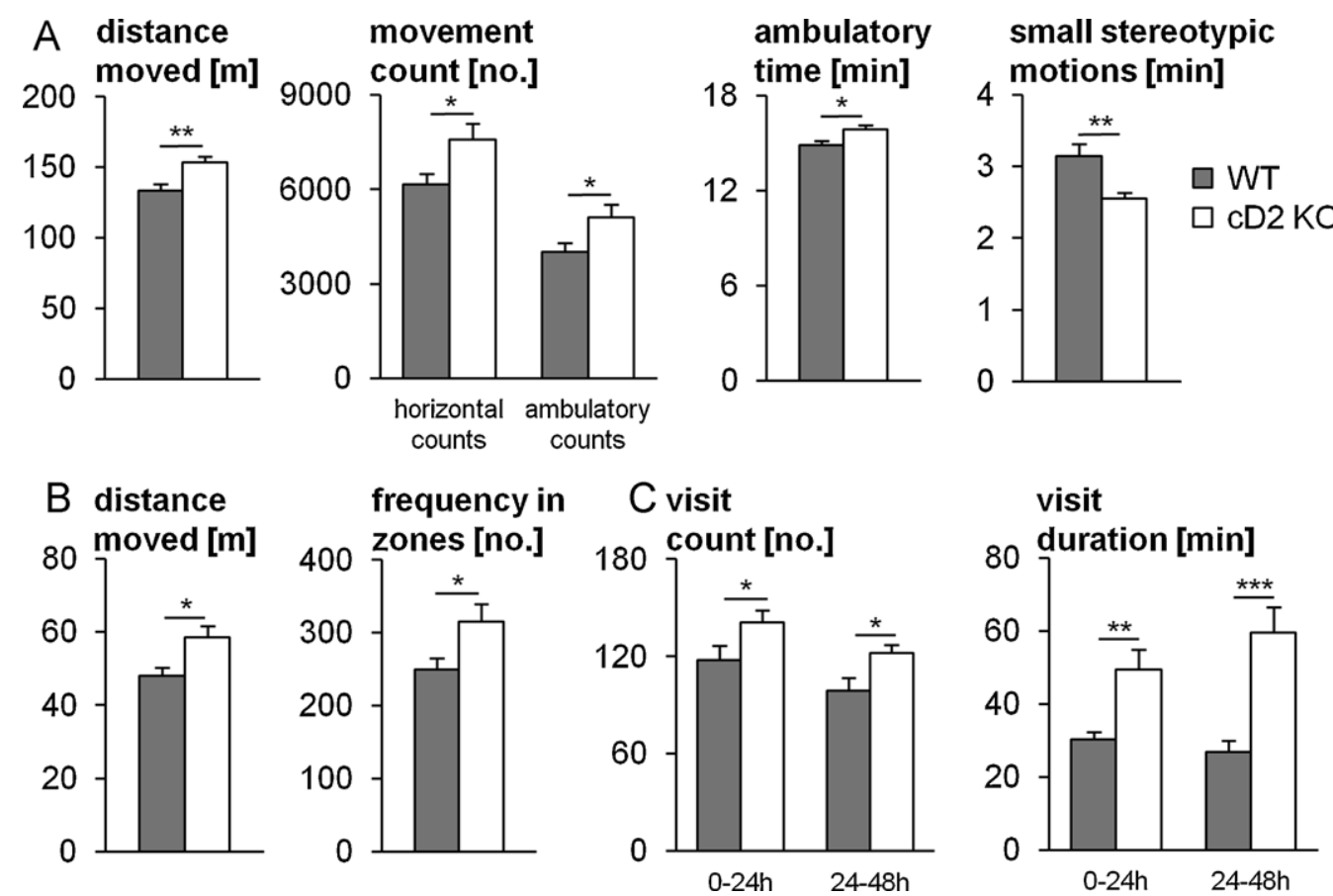

Fig. 3. Mutant mice showed increased activity when compared to WT controls in automated motility chamber, open field, and IntelliCage automated system. (A) In the automated motility chamber, cD2 KO mutants travelled longer distances, performed more horizontal and ambulatory movements, and showed increased ambulatory movement time comparing to WT controls. Moreover, cD2 KO mice showed shorter time of small stereotypic motions than control animals. (B) In the open field, cD2 KO mice travelled longer distances and showed higher frequency of presence in all the zones of the arena than WT animals. (C) In the IntelliCage automated system, cD2 KO mice were more active than WT controls what was reflected by more frequent and longer visits in the corners during $48 \mathrm{~h}$ of observation. The results are presented as means \pm S.E.M.; $\left({ }^{*}\right) P<0.05,\left({ }^{* *}\right) P<0.01,\left({ }^{* * *}\right) P<0.001$. 


\section{Discussion}

\subsection{Evaluation of the mouse model}

We have employed cD2 KO mice with a new genetic background, i.e., following multiple backcrossing with animals from C57BL/6 strain. The new mutant mice showed yet again all the main features of formerly used cD2 KO mice of different, mixed genetic background [1,2]. Namely, they had reduced, possibly eliminated, adult brain neurogenesis, reduced hippocampal size and efficient learning in fear conditioning paradigm. Since it was shown repeatedly that the genetic background can influence phenotype of transgenic mice (summarized during Banbury Conference [15]), repeating our previous results using CD2 KO mutation in new genetic background, both strengthens the importance of cyclin D2 involvement in adult brain neurogenesis and its irrelevance for hippocampal learning.

We have shown previously [1] that genetic impairment of adult brain neurogenesis in $\mathrm{cD} 2 \mathrm{KO}$ mice does not influence learning or memory formation. The mice learned several behavioral tasks like context fear conditioning, trace fear conditioning, novel object recognition, the hidden-platform version of the Morris water maze, and spatial tests in IntelliCages surprisingly well. The major finding of unimpaired cue and context fear conditioning was reproduced herein using $\mathrm{cD} 2 \mathrm{KO}$ with a novel genetic background.

Some investigators suggested the role of new neurons in context fear conditioning [e.g., 12], others - did not report their importance [e.g., 16, 17]. However, hippocampally lesioned mice and rats were repeatedly shown to be impaired in learning and memory models, particularly in those involving spatial components [e.g., 18]. Such animals were also shown to be impaired in several species-typical behaviors [e.g., 4]. Hence, we aimed at verifying, whether CD2 KO mice with reduced hippocampal formation and highly reduced adult brain neurogenesis were also impaired in these behaviors.

\subsection{Main finding and its limitations}

In general, cD2 KO mice with impaired adult brain neurogenesis and smaller hippocampal formation showed significant deficits in non-learning behaviors, typical for mice with hippocampal lesions, while preserving hippocampal-dependent learning. These nonlearning behaviors included nest building, digging, marble burying, delayed sucrose preference and increased activity.

We realize, our model, approach and conclusions have limitations. In our present study and in earlier reports [1,2], we found that cD2 KO mice have smaller brains overall, i.e., the hippocampal formation is reduced but smaller are also other brain regions like neocortex and cerebellum. It is hence possible that disturbances in other brain regions, not only the hippocampal formation, or a generally disturbed neurocircuitry might account for the observed impairments in species-typical behaviors. For example, it was shown by others that medial prefrontal cortex-lesioned mice exhibit higher locomotor activity than that of the controls [19-21]. However, this procedure did not impair nesting behavior in mice [19]. We would still argue that the collection of paradigms which were impaired in cD2 KO mice closely resembles that of hippocampally lesioned animals. However, it is more specific - impaired are non-learning paradigms while learning itself, affected in lesioned mice, seems to be spared. It is also possible, that species-typical behaviors we employed here, are more sensitive to genetic manipulations in mice than aversive classical conditioning we used in this work as well as other learning and memory paradigms we used before [1].

\subsection{Species-typical behaviors}

Cyclin D2 KO mice built none or poorer nests. Notably, nesting impairment was observed in mice, rats, and gerbils with hippocampal damage [3,4,22-24]. Furthermore, mutant mice were much slower than the WT controls to start digging and spent much less time digging in total, and performing less digging bouts. Such behavior was observed before in mice with hippocampal lesions [6]. Similarly, cD2 KO mice had reduced burying behavior and were hiding less marbles. Again, parallel results were obtained in mice with hippocampal lesions [6].

\subsection{Delayed sucrose preference}

Mutant mice showed no signs of preference for sweetened water during the first session and developed the preference during the second exposition to the sucrose solution. Interestingly, rats with hippocampal lesions failed to show any preference for sucrose in the 5 min test session, whereas the same animals were identical to control subjects in the $24 \mathrm{~h}$ test [25]. The delayed preference could have several possible explanations. In general, we observe that cD2 KO mice are initially less active when exploring a novel environment and increase their activity as time progresses (see below). They take time to start to explore, it takes them more time to get familiar with the novel surroundings, and only later they become more active then controls. This could explain why also sucrose preference appeared later in the mutant mice. Moreover, cD2 KO mice have impaired olfaction [1]. As a result, they may take longer to distinguish between both tastes and, in consequence, their olfactory/gustatory learning could be also impaired.

Reduced size of hippocampal formation and whole brain grey matter was observed in patients with major depression [26] and bipolar disorder [e.g., 27]. Hence, one might also expect cD2 KO mice to demonstrate, e.g., depression-like behaviors. However, we did not detect such behaviors (not published), also there is little probability that delayed preference of sucrose is a sign of anhedonia or neophobia which are regarded as depression-like behaviors. Mice did drunk as much sucrose solution as water initially and the lack of preference did not persist. Finally, a far-fetched explanation could be that cD2 KO mice show attention-deficit disorder-like symptoms with increased activity and lack of attention for sucrose; similar symptoms were reported in trkB receptor knockout mice [28].

\subsection{Motor and activity tests}

Mutant and WT mice did not differ on the accelerating rotarod. Similar lack of difference in rotarod performance was observed before in hippocampally lesioned mice [4]. However, cD2 KO mice were more active when compared with WT mice in the automated motility chamber, in the open field, and in IntelliCage, which again paralleled the previous results obtained with hippocampally lesioned mice [e.g., 4]. Interestingly, most of the differences between $\mathrm{CD} 2 \mathrm{KO}$ and WT mice were observed later in time, e.g., increased activity in IntelliCage was not observed after $12 \mathrm{~h}$ but was significant following $24 \mathrm{~h}$ of observation (not shown). Such results are in line with the previous observation of delayed initiation of behavior in hippocampally lesioned mice. Moreover, in tests where these mice experienced the same or similar environments repeatedly, or over a long period, activity tended to be higher than in the WT controls [4]. As mentioned above the delayed initiation of behavior of mutant mice might also explain for apparent initial lack of preference in sucrose drinking.

We observed a decrease in small stereotypic movement in cD2 KO mice, whereas previous lesion studies showed increased stereotypic behavior in the animals with hippocampal lesions [e.g., 
29] or dentate gyrus lesions [e.g., 30]. This apparent discrepancy appeared because we detected only small stereotypic movements, like scratching, digging and grooming, while large stereotypic motions like impulsive jumping and running, pronounced in hippocampally lesioned animals, was not detected. Also, there was no difference in stereotypic movements bursts between WT and cD2 KO mice.

\subsection{Investigated behaviors and adult brain neurogenesis}

There is a limited literature connecting species-typical behaviors with adult brain neurogenesis. In particular, mice where the proliferation of new neurons was inhibited through conditional deletion of ataxia telangeictasia-mutated and rad-3 related gene (ATR), coding for cell cycle checkpoint kinase, showed significant reduction in the number of marbles buried during a 15 min session [31]. However, marble burying is mainly employed as a measure of anxiety in the context of neurogenesis [31,32, see below]. Finally, to the best of our knowledge there have been no data available on the impact of adult brain neurogenesis on other species-typical behaviors like digging or nest building.

Results of studies relating adult brain neurogenesis and changes in activity are both complex and somewhat contradictory. In most of the cases, reduction of adult neurogenesis was shown to have no influence on overall activity of animals [e.g., 16, 33-36]. Notably, however, the measurements of activity were limited to shorttime one-time-only tests. Yet in several cases, transgenic animals with impaired adult brain neurogenesis showed increased activity. For example glucose-dependent insulinotropic polypeptide receptor knockout mice with impaired neurogenesis had increased open field activity [37]. Also, FoxG1 haploinsufficiency resulted in impaired neurogenesis and hyperlocomotion [38]. However, lower neurogenesis was found to be associated with higher anxiety (see below) and depressive-like behaviors linked, in turn, with lower activity [e.g., 39]. On the other hand, physical activity increases neurogenesis $[40,41]$, moreover, several recent findings suggest that running-induced neurogenesis increases anxiety in mice and rats [42].

Similarly, the relation between adult brain neurogenesis and anxiety deserves separate comment. Most studies show that altering levels of adult neurogenesis does not influence the levels of anxiety in mice and rats [e.g., 12, 43]. However, several recent reports have suggested a connection between adult neurogenesis and anxiety-related behaviors. Transgenic animals with overexpression of the pro-apoptotic protein Bax in neuronal precursors and impaired adult hippocampal neurogenesis exhibited a striking increase in anxiety-related behaviors in the elevated plus maze and in the light/dark emergence test as well as in the presence predator exposure test [44]. Moreover, mice overexpressing follistatin with reduced survival of new neurons exhibited enhanced anxiety in the open field, light-and-dark choice test, and novel-area accessing test [45]. Also, a remarkably increased anxiety-like behavior in the open field and elevated plus-maze was observed in mice carrying TrkB deletion affecting integration of new neurons into hippocampal circuits [46]. However, others have postulated that adult hippocampal neurogenesis is a crucial player in the genesis of anxiety. Fuss et al. found that voluntary running induced increased anxiety in the open field, elevated O-maze, and dark-light-box accompanied by a significant increase of hippocampal neurogenesis [47]. Moreover, studies from rodents with hippocampal lesions showed decrease in anxiety levels [e.g., 6].

The increased activity and reduced number of marbles buried could also be related to a reduction in anxiety. However (see above), the relation between the levels of new neurons and anxiety is controversial. Also, the validity of marble burying assay as an adequate animal model of anxiety behavior was questioned [e.g., 48].
However, we cannot rule out the possibility that changes in anxiety also affected the results presented in this work, though we did not observe changed anxiety levels in CD2 KO mice before [1].

\section{Conclusion}

The functional significance of the adult brain neurogenesis remains unclear, although it has been repeatedly suggested to play a role in learning processes and memory formation. Aiming to establish the exact function of new neurons of the dentate gyrus, most of the investigators are ablating these cells in order to find out the resulting deficits. However, removing newly formed cells most likely results in both eliminating new neurons as well as affecting the hippocampal connectivity and volume as well. This makes it impossible to distinguish between the behavioral results of impaired neurogenesis and impaired functioning of hippocampal formation.

In cD2 KO mice, the ablation of the adult brain neurogenesis is permanent throughout the adult life and, therefore, is not associated with abruptly disturbed hippocampal connectivity. As a result, cD2 KO mice were shown not to be deficient in several hippocampal-dependent learning paradigms. However, most likely due to the permanent lack of cyclin D2, also during the development, the volume of the hippocampal formation is substantially smaller in these mice [2]. Therefore, we investigated whether these mice showed any signs of impaired hippocampal functioning. The animals turned out to be deficient in several species-natural behaviors and showed increased mobility - the results observed also following the hippocampal lesions. These results suggest that the impaired neurogenesis, impaired hippocampal circuitry and smaller hippocampal formation could have separate behavioral outcomes. Such conclusion should have consequences for researchers investigating the behavioral impact of disturbed adult hippocampal neurogenesis.

Morphological abnormalities of the hippocampal formation and/or impairment in adult brain neurogenesis in cD2 KO mice alter their hippocampal-dependent behaviors typical for small rodents. On the other hand, these mice showing lack of adult neurogenesis display normal learning and memory in different hippocampally dependent learning paradigms [1]. Together, these results suggest that adult neurogenesis may play an important role in several hippocampal-dependent tasks rather than learning. In conclusion, the results from the present study support a role for the hippocampal formation in species-typical behaviors and suggest a key role of immature neurons in these behaviors, a role which might be linked with their specific and unique features.

\section{Acknowledgement}

This work was supported by the European Union structural funds - Innovative Economy Operational Program - Project No. POIG.01.01.02-00-109/09.

\section{References}

[1] Jaholkowski P, Kiryk A, Jedynak P, Ben Abdallah NM, Knapska E, Kowalczyk A, et al. New hippocampal neurons are not obligatory for memory formation; cyclin D2 knockout mice with no adult brain neurogenesis show learning. Learning \& Memory 2009;16:439-51.

[2] Kowalczyk A, Filipkowski RK, Rylski M, Wilczynski GM, Konopacki FA, Jaworski $\mathrm{J}$, et al. The critical role of cyclin D2 in adult neurogenesis. Journal of Cell Biology 2004;167:209-13.

[3] Antonawich FJ, Melton CS, Wu P, Davis JN. Nesting and shredding behavior as an indicator of hippocampal ischemic damage. Brain Research 1997;764:249-52.

[4] Deacon RM, Croucher A, Rawlins JN. Hippocampal cytotoxic lesion effects on species-typical behaviours in mice. Behavioural Brain Research 2002;132:203-13.

[5] Deacon RM, Raley JM, Perry VH, Rawlins JN. Burrowing into prion disease. Neuroreport 2001;12:2053-7. 
[6] Deacon RM, Rawlins JN. Hippocampal lesions, species-typical behaviours and anxiety in mice. Behavioural Brain Research 2005;156:241-9.

[7] Guenther K, Deacon RM, Perry VH, Rawlins JN. Early behavioural changes in scrapie-affected mice and the influence of dapsone. The European Journal of Neuroscience 2001;14:401-9.

[8] Good M, Honey RC. Dissociable effects of selective lesions to hippocampal subsystems on exploratory behavior, contextual learning, and spatial learning. Behavioral Neuroscience 1997; 111:487-93.

[9] Sicinski P, Donaher JL, Geng Y, Parker SB, Gardner H, Park MY, et al. Cyclin D2 is an FSH-responsive gene involved in gonadal cell proliferation and oncogenesis. Nature 1996;384:470-4.

[10] Deacon RM. Burrowing in rodents: a sensitive method for detecting behaviora dysfunction. Nature Protocols 2006;1:118-21.

[11] Knapska E, Maren S. Reciprocal patterns of c-Fos expression in the medial prefrontal cortex and amygdala after extinction and renewal of conditioned fear. Learning \& Memory 2009;16:486-93.

[12] Saxe MD, Battaglia F, Wang JW, Malleret G, David DJ, Monckton JE, et al. Ablation of hippocampal neurogenesis impairs contextual fear conditioning and synaptic plasticity in the dentate gyrus. Proceedings of the National Academy of Sciences of the United States of America 2006;103:17501-6.

[13] Deacon RM. Digging and marble burying in mice: simple methods for in vivo identification of biological impacts. Nature Protocols 2006;1:122-4.

[14] Kalueff AV, Keisala T, Minasyan A, Kuuslahti M, Miettinen S, Tuohimaa P. Behavioural anomalies in mice evoked by Tokyo disruption of the vitamin $\mathrm{D}$ receptor gene. Neuroscience Research 2006;54:254-60.

[15] Banbury. Mutant mice and neuroscience: recommendations concerning genetic background. Banbury Conference on genetic background in mice. Neuron 1997; 19:755-9.

[16] Shors TJ, Townsend DA, Zhao M, Kozorovitskiy Y, Gould E. Neurogenesis may relate to some but not all types of hippocampal-dependent learning. Hippocampus 2002;12:578-84.

[17] Zhang CL, Zou Y, He W, Gage FH, Evans RM. A role for adult TLX-positive neural stem cells in learning and behaviour. Nature 2008:451:1004-7.

[18] Deacon RM, Bannerman DM, Kirby BP, Croucher A, Rawlins JN. Effects of cytotoxic hippocampal lesions in mice on a cognitive test battery. Behavioural Brain Research 2002;133:57-68.

[19] Deacon RM, Penny C, Rawlins JN. Effects of medial prefrontal cortex cytotoxic lesions in mice. Behavioural Brain Research 2003;139:139-55.

[20] Lacroix L, Broersen LM, Weiner I, Feldon J. The effects of excitotoxic lesion of the medial prefrontal cortex on latent inhibition, prepulse inhibition, food hoarding, elevated plus maze, active avoidance and locomotor activity in the rat. Neuroscience 1998;84:431-42.

[21] Yee BK. Cytotoxic lesion of the medial prefrontal cortex abolishes the partial reinforcement extinction effect, attenuates prepulse inhibition of the acoustic startle reflex and induces transient hyperlocomotion, while sparing spontaneous object recognition memory in the rat. Neuroscience 2000;95:675-89.

[22] Glickman SE, Higgins TJ, Isaacson RL. Some effects of hippocampal lesions on the behavior of Mongolian gerbils. Physiology \& Behavior 1970;5:931-8.

[23] Kim C. Nest building, general activity, and salt preference of rats following hippocampal ablation. Journal of Comparative and Physiological Psychology 1960;53:11-6.

[24] Kimble DP, Rogers L, Hendrickson CW. Hippocampal lesions disrupt maternal, not sexual, behavior in the albino rat. Journal of Comparative and Physiological Psychology 1967;63:401-7.

[25] Murphy HM, Brown TS. Effects of hippocampal lesions on simple and preferential consummatory behavior in the rat. Journal of Comparative and Physiological Psychology 1970;72:404-15.

[26] Bremner JD, Narayan M, Anderson ER, Staib LH, Miller HL, Charney DS. Hippocampal volume reduction in major depression. The American Journal of Psychiatry 2000;157:115-8.

[27] Brown GG, Lee JS, Strigo IA, Caligiuri MP, Meloy MJ, Lohr J. Voxel-based morphometry of patients with schizophrenia or bipolar I disorder: a matched control study. Psychiatry Research 2011;194:149-56.
[28] Zorner B, Wolfer DP, Brandis D, Kretz O, Zacher C, Madani R, et al. Forebrainspecific trkB-receptor knockout mice: behaviorally more hyperactive than depressive. Biological Psychiatry 2003;54:972-82.

[29] Kimble DP. The effects of bilateral hippocampal lesions in rats. Journal of Comparative and Physiological Psychology 1963;56:273-83.

[30] Mickley GA, Ferguson JL, Nemeth TJ, Mulvihill MA, Alderks CE. Spontaneous perseverative turning in rats with radiation-induced hippocampal damage. Behavioral Neuroscience 1989;103:722-30.

[31] Onksen JL, Brown EJ, Blendy JA. Selective Deletion of a cell cycle checkpoint kinase (ATR) reduces neurogenesis and alters responses in rodent models of behavioral affect. Neuropsychopharmacology 2011;36:960-9.

[32] Dagyte G, Crescente I, Postema F, Seguin L, Gabriel C, Mocaer E, et al. Agomelatine reverses the decrease in hippocampal cell survival induced by chronic mild stress. Behavioural Brain Research 2011;218:121-8.

[33] Kitamura T, Saitoh Y, Takashima N, Murayama A, Niibori Y, Ageta H, et al. Adult neurogenesis modulates the hippocampus-dependent period of associative fear memory. Cell 2009;139:814-27.

[34] Rola R, Raber J, Rizk A, Otsuka S, VandenBerg SR, Morhardt DR, et al. Radiationinduced impairment of hippocampal neurogenesis is associated with cognitive deficits in young mice. Experimental Neurology 2004;188:316-30.

[35] Saxe MD, Malleret G, Vronskaya S, Mendez I, Garcia AD, Sofroniew MV, et al. Paradoxical influence of hippocampal neurogenesis on working memory. Proceedings of the National Academy of Sciences of the United States of America 2007; 104:4642-6.

[36] Shors TJ, Miesegaes G, Beylin A, Zhao M, Rydel T, Gould E. Neurogenesis in the adult is involved in the formation of trace memories. Nature 2001;410:372-6.

[37] Faivre E, Gault VA, Thorens B, Holscher C. Glucose-dependent insulinotropic polypeptide receptor knockout mice are impaired in learning, synaptic plasticity, and neurogenesis. Journal of Neurophysiology 2011.

[38] Shen L, Nam HS, Song P, Moore H, Anderson SA. FoxG1 haploinsufficiency results in impaired neurogenesis in the postnatal hippocampus and contextual memory deficits. Hippocampus 2006;16:875-90.

[39] Santarelli L, Saxe M, Gross C, Surget A, Battaglia F, Dulawa S, et al. Requiremen of hippocampal neurogenesis for the behavioral effects of antidepressants. Science 2003;301:805-9.

[40] van Praag H, Christie BR, Sejnowski TJ, Gage FH. Running enhances neurogenesis, learning, and long-term potentiation in mice. Proceedings of the National Academy of Sciences of the United States of America 1999;96:13427-31.

[41] van Praag H, Kempermann G, Gage FH. Running increases cell proliferation and neurogenesis in the adult mouse dentate gyrus. Nature Neuroscience 1999;2:266-70.

42] Fuss J, Ben Abdallah NM, Hensley FW, Weber KJ, Hellweg R, Gass P. Deletion of running-induced hippocampal neurogenesis by irradiation prevents development of an anxious phenotype in mice. PloS One 2010;5:e12769.

[43] Meshi D, Drew MR, Saxe M, Ansorge MS, David D, Santarelli L, et al. Hippocampal neurogenesis is not required for behavioral effects of environmental enrichment. Nature Neuroscience 2006;9:729-31.

[44] Revest JM, Dupret D, Koehl M, Funk-Reiter C, Grosjean N, Piazza PV, et al. Adult hippocampal neurogenesis is involved in anxiety-related behaviors. Molecular Psychiatry 2009;14:959-67.

[45] Ageta H, Murayama A, Migishima R, Kida S, Tsuchida K, Yokoyama M, et al Activin in the brain modulates anxiety-related behavior and adult neurogenesis. PloS One 2008;3:e1869.

[46] Bergami M, Rimondini R, Santi S, Blum R, Gotz M, Canossa M. Deletion of TrkB in adult progenitors alters newborn neuron integration into hippocampal circuits and increases anxiety-like behavior. Proceedings of the National Academy of Sciences of the United States of America 2008;105:15570-5.

[47] Fuss J, Ben Abdallah NM, Vogt MA, Touma C, Pacifici PG, Palme R, et al. Voluntary exercise induces anxiety-like behavior in adult C57BL/6J mice correlating with hippocampal neurogenesis. Hippocampus 2010;20:364-76.

[48] Jimenez-Gomez C Osentoski A, Woods JH. Pharmacological evaluation of the adequacy of marble burying as an animal model of compulsion and/or anxiety. Behavioural Pharmacology 2011;22:711-3. 